\title{
The collisional atomic processes in geo-cosmical plasmas: data needed for spectroscopy
}

\author{
V.A. Srećković ${ }^{1}$, Lj.M. Ignjatović ${ }^{1}$, M.S. Dimitrijević ${ }^{2,3}$, \\ N.N. Bezuglov ${ }^{4}$ and A.N. Klyucharev ${ }^{4}$ \\ 1 Institute of Physics Belgrade, BU, Pregrevica 118, 11080 Belgrade, Serbia \\ (E-mail:vlada@ipb.ac.rs) \\ 2 Astronomical Observatory, Volgina 7, 11060 Belgrade, Serbia \\ 3 Sorbonne Université, Observatoire de Paris, Université PSL, CNRS, \\ LERMA, F-92190, Meudon, France (E-mail: mdimitrijevic@aob.rs) \\ 4 Department of Physics, Saint Petersburg State University, 7/9 \\ Universitetskaya nab., 199034 St. Petersburg, Russia
}

Received: July 16, 2019; Accepted: September 29, 2019

\begin{abstract}
The rate coefficients for the chemi-ionization (CI) processes in $\mathrm{Na}^{*}(n)+\mathrm{Na}, \mathrm{Li}^{*}(n)+\mathrm{Na}, \mathrm{Li}^{*}(n)+\mathrm{Li}$ and $\mathrm{H}^{*}(n)+\mathrm{Li}$ collisions are calculated for wide region of temperatures and the principal quantum numbers. The presented values of the rate coefficients are very useful for the improvement of modelling and analysis of different layers of weakly ionized plasmas in atmospheres of various stars where these and other CI processes could be important and could change the optical characteristics. Also, the results are of interest in spectroscopy of low temperature laboratory plasma.
\end{abstract}

Key words: Atomic processes - Planets and satellites: general - Line: profiles - Plasmas- ISM: clouds

\section{Introduction}

Collisional processes which include atoms and molecules in geo-cosmical plasmas, constantly attract attention of scientists (see e.g. Chary et al., 1999; Klyucharev et al., 2007). Among these processes, those involving highly excited Rydberg atoms are of particular interest (Gnedin et al., 2009; O'Keeffe et al., 2012). Primarily this can be attributed to a group of chemi-ionization (CI) processes in Rydberg atom collisions with ground state parent atoms. In a series of papers (Mihajlov et al., 2008; Mihajlov et al., 2011a; Mihajlov et al., 2011b; Mihajlov et al., 2016; Srećković et al., 2018), the group of atom-Rydberg atom collisional processes involving hydrogen and helium have been studied from the point of view of their effect on the optical (spectral line shapes) and kinetic properties of weakly ionized laboratory and astrophysical plasmas.

In this contribution we investigate CI processes for astrophysical usage for the case of alkali metals and give results which enable modeling of geo-cosmic weakly ionized plasma for wide range of plasma parameters. The obtained CI 
data are needed for modeling and research of cool stars, like white and brown dwarfs (Debes et al., 2012; Xu \& Jura, 2014), lithium stars (Shavrina et al., 2001, 2003), sodium clouds around Io (Mendillo et al., 1990; Wilson et al., 2002), cometary tails (Cremonese et al., 2002, 1997), and primordial gas containing Li atoms and ions (Gnedin et al., 2009; Coppola et al., 2011), etc. Also, study of the CI collisional processes of alkali atoms are of basic interest for some laboratory spectroscopical investigation and technical applications (Vujčić et al., 2015; Efimov et al., 2017; Marinković et al., 2017; Pichler et al., 2017; Beuc et al., 2018a,b).

In Section 2 we describe the methods of calculation and then in Section 3 we give the results of the calculation. In Section 4 the conclusions are presented.

\section{Theoretical remarks}

\subsection{The method of calculations}

We will consider two types of CI processes: the symmetric processes

$$
A^{*}(n)+A \Rightarrow e+\left\{\begin{array}{l}
A_{2}^{+}, \\
A+A^{+},
\end{array}\right.
$$

and the non-symmetric processes

$$
A^{*}(n)+B \Rightarrow e+\left\{\begin{array}{l}
A B^{+}, \\
A+B^{+}
\end{array}\right.
$$

where $A, B, A^{+}$and $B^{+}$are atoms and ions in the ground states, $A^{*}(n)$ is highly excited atom i.e. Rydberg atom, $A_{2}^{+}$and $A B^{+}$are the molecular ions in the ground electronic states. In Eq. (2) $B$ atoms ionization potential is less than $A$ ionization potential. The CI processes (1) and (2) can be divided to the associative ionization channel (a) with formation of molecular ions $A_{2}^{+}$or $A B^{+}$ and to the non-associative ionization channel (b).

These processes have already been treated on the basis of the so-called dipole resonant mechanism (DRM), so we will briefly describe its main features. In this description of collisional ionisation and excitation events, these processes are induced by the dipole part of the electrostatic interaction between the outer Rydberg electron and the inner ion-atom system (see Fig. 1). A detailed description of the mechanism can be found in Mihajlov et al. (2012). 


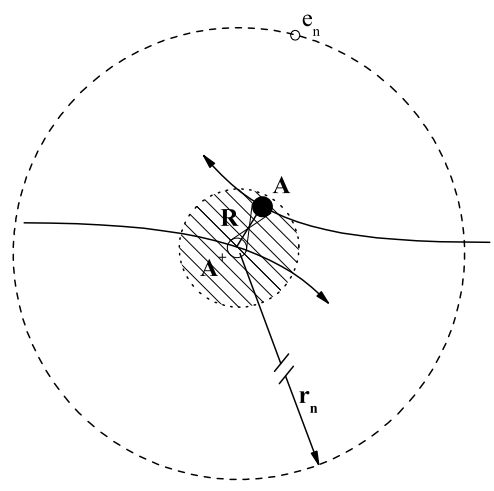

Figure 1. Schematic illustration of RA $+\mathrm{A}$ collision. Here $R$ is the internuclear distance in the collision system, $r_{n} \sim n^{2}$ is the characteristic radius of Rydberg's atom $A^{*}, e_{n}$ is outer electron and $A$ and $A^{+}$are atoms and their positive ions.

\subsection{The CI rate coefficients}

In accordance with the CI theory the partial rate coefficients $K^{(a)}(n, T)$ and the total ones $K^{(a b)}(n, T)$ can be presented with relations

$K^{(a)}(n, T)=\int_{0}^{E_{m}^{(a)}(n)} v \sigma^{(a)}(n, E) f(v ; T) d v, \quad K^{(a b)}(n, T)=\int_{0}^{\infty} v \sigma^{(a b)}(n, E) f(v ; T) d v$

where the cross-sections $\sigma^{(a, a b)}(n, E)$ are given in Mihajlov et al. (2012), $E_{m}^{(a)}(n)$ is the upper limit of $E$ relevant for the associative ionization process (1a) or (2a), and $f(v ; T)=f_{\text {cell }}(v ; T)$ is Maxwell distribution function. The rate coefficient $K^{(b)}(n, T)$ for the process (b) is obtained from $K^{(a b)}(n, T)=K^{(a)}(n, T)+$ $K^{(b)}(n, T)$.

For understanding plasma chemistry and improvement of modelling and analysis of different layers of weakly ionized plasmas in atmospheres of various stars the cross sections and rate coefficients for reactions are needed (Mihajlov et al., 2011c; Srećković et al., 2017, 2018). In this contribution we investigate CI processes for astrophysical usage and give results which enable modeling of geo-cosmic weakly ionized plasma. 


\section{Results and Applications}

\subsection{The calculated quantities}

In accordance with the described method, we calculate the rate coefficients $K^{(a b)}(n, T)$ for the extended range of principal quantum numbers $n \leq 25$ and temperatures $500 \mathrm{~K} \leq T \leq 1500 \mathrm{~K}$ for the CI processes in $\mathrm{Li}^{*}(n)+\mathrm{Na}$,

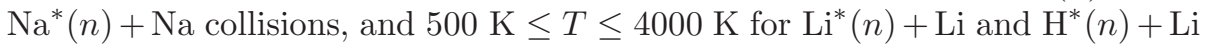
collisions in order to use in modelling and analysis of different layers of weakly ionized plasmas in atmospheres of various stars. We have extended the range of temperatures up to $4000 \mathrm{~K}$ for lithium to enable the possible inclusion of CI processes in modelling of cool stars with low effective temperatures and enormous high Li abundances.

We note that the rate coefficient $K^{(a b)}(n, T)\left(\right.$ for $\mathrm{Li}^{*}(n)+\mathrm{Li}, \mathrm{Li}^{*}(n)+\mathrm{Na}$, and $\mathrm{Na}^{*}(n)+\mathrm{Na}$ ) has the maximum between $n=6$ and $n=10$. The maximum position depend on temperature and moves to lower values of $n$ with the increase of the temperature. A different behavior of $K^{(a b)}(n, T)$ is for the case of $\mathrm{H}^{*}(n)+\mathrm{Li}$ where the rate coefficient decreases with the increase of $n$, with different dependence on temperature. In the regions of lower $T$ the process of associative CI, i.e. creation of molecular ions, in (1a) or (2a) collisions dominate in comparison with the non-associative CI channel except for the case of $\mathrm{H}^{*}(n)+\mathrm{Li}$ collisions where the associative channel is totally negligible for the analyzed conditions.

\subsection{Fitting formula}

In astrophysics, especially for the modeling of stellar atmospheres, fitting formula and coefficients as functions of temperature are needed, since such fitting coefficients are easier to be imported to the codes for stellar atmosphere modeling than tabulated data for a set of temperatures. To enable easy and more adequate use in laboratory as well as to allow a faster computation in stellar atmospheres modeling we construct a fitting formula. The results are in the form of a simple fitting formula:

$$
\log \left(K^{(a b)}(n ; T)\right)=k_{1}(n)+k_{2}(n) \cdot \log (T)+k_{3}(n) \cdot(\log (T))^{2} .
$$

In Tabs. 1 and 2 the selected fits for $4 \leq n \leq 25$ are listed for the total rate coefficients of CI processes (1) and (2). It should be noted that fits in Tab. 1 are valid within the temperature range $500 \mathrm{~K} \leq T \leq 1500 \mathrm{~K}$ for $\mathrm{Li}^{*}(n)+\mathrm{Na}$, $\mathrm{Na}^{*}(n)+\mathrm{Na}$ collisions and in Tab. 2 are valid within the temperature range $500 \mathrm{~K} \leq T \leq 4000 \mathrm{~K}$ for $\mathrm{Li}^{*}(n)+\mathrm{Li}$ and $\mathrm{H}^{*}(n)+\mathrm{Li}$ collisions. 
Table 1. The fits to the rate coefficient. The fits were made to the results over the range $500 \mathrm{~K} \leq T \leq 1500 \mathrm{~K}$.

\begin{tabular}{cccccccc}
\hline & \multicolumn{3}{c}{$\mathrm{Li}^{*}(n)+\mathrm{Na}$} & & \multicolumn{3}{c}{$\mathrm{Na}^{*}(n)+\mathrm{Na}$} \\
\cline { 2 - 4 } \cline { 6 - 8 } $\mathrm{n}$ & $\mathrm{k} 1$ & $\mathrm{k} 2$ & $\mathrm{k} 3$ & & $\mathrm{k} 1$ & $\mathrm{k} 2$ & $\mathrm{k} 3$ \\
\hline \hline 4 & -41.35531 & 18.67062 & -2.69225 & & -46.51493 & 21.4718 & -3.10971 \\
5 & -16.97267 & 4.48624 & -0.58834 & -29.28982 & 11.44201 & -1.62003 \\
10 & -14.06446 & 2.72686 & -0.34263 & -11.58289 & 1.24937 & -0.11571 \\
15 & -15.367 & 3.08165 & -0.40038 & -9.68059 & 0.23985 & 0.00292 \\
20 & -15.80875 & 2.96859 & -0.38137 & & -9.50371 & 0.07705 & 0.00612 \\
22 & -15.8578 & 2.86113 & -0.36295 & & -9.31387 & -0.10334 & 0.03144 \\
25 & -16.2462 & 2.93717 & -0.37582 & & -9.38528 & -0.14156 & 0.03217
\end{tabular}

Table 2. Same as in Tab.1 but over the temperature range $500 \mathrm{~K} \leq T \leq 4000 \mathrm{~K}$.

\begin{tabular}{cccccccc}
\hline & \multicolumn{3}{c}{$\mathrm{Li}^{*}(n)+\mathrm{Li}$} & & \multicolumn{3}{c}{$\mathrm{H}^{*}(n)+\mathrm{Li}$} \\
\cline { 2 - 4 } \cline { 6 - 7 } $\mathrm{n}$ & $\mathrm{k} 1$ & $\mathrm{k} 2$ & $\mathrm{k} 3$ & & $\mathrm{k} 1$ & $\mathrm{k} 2$ & $\mathrm{k} 3$ \\
\hline \hline 4 & -35.12844 & 14.13288 & -1.89755 & & -5.79278 & -1.1651 & 0.13442 \\
5 & -23.31012 & 7.66745 & -0.99756 & -6.22763 & -1.12647 & 0.12474 \\
10 & -11.29713 & 1.25562 & -0.12888 & -7.78576 & -1.05661 & 0.11177 \\
15 & -9.71227 & 0.36138 & -0.0305 & -8.67836 & -1.04816 & 0.11043 \\
20 & -9.45545 & 0.06564 & 0.00121 & -9.23865 & -1.08876 & 0.11676 \\
22 & -9.46101 & 0.00324 & 0.00775 & -9.46812 & -1.07506 & 0.11468 \\
25 & -9.5051 & -0.07041 & 0.01609 & -9.75651 & -1.06821 & 0.11363
\end{tabular}

\subsection{Astrophysical Targets}

\subsubsection{Li-rich stars}

Study of CI processes may be of interest for the investigation and modelling of plasma in cold lithium stars (see North et al., 1998; Shavrina et al., 2001, 2003). Recently Li et al. (2018) found high-lithium abundance in newly discovered 12 low-mass, metal-poor, main-sequence stars, and in red giant stars in the Milky Way halo. Collisional processes including CI could be of interest for studying of lithium-rich stellar atmospheres as an additional canal for the creation of Li I atoms. The considered CI processes influence as well the ionization level and atom excited-state populations, so that they could affect the optical properties of the weakly ionized regions of alkali rich plasmas.

As it is known lithium is easily ionized and in the spectra of cool stars the Li I resonance line $6707.8 \AA$ is commonly observed. The lithium abundance data in such stars are mostly acquired from the spectral analysis of this particular line. The investigated CI processes, which influence atom excited-state populations and the ionization level, influence the optical properties and could be important 
for the lithium spectra. Consequently the CI processes could be important for atmospheres of lithium-rich stars as an supplementary channel for the creation of the lithium atoms and RA atom populations. We give the data for extended range of temperatures for the calculation for lithium (see Tab. 2) to enable the possible inclusion of CI processes in $\mathrm{Li}^{*}(n)+\mathrm{Li}$ and $\mathrm{H}^{*}(n)+\mathrm{Li}$ collisions in modeling of cool stars with low effective temperatures $\left(T_{\text {eff }} \leq 6000 \mathrm{~K}\right)$ and enormous high Li abundances (Li et al., 2018).

\subsubsection{Primordial gas}

It is known that in the early Universe the chemical composition of the primordial gas contained lithium, and during the cooling of Universe there is an epoch when conditions for presently investigated RA collisional processes were favorable (Gnedin et al., 2009; Puy et al., 2007). Collisional recombination, ionization and Rydberg states can play important role for the early Universe chemistry (Coppola et al., 2011). As concluded in (Coppola et al., 2011; Puy et al., 2007) $\mathrm{LiH}^{+}$represents the most abundant molecular species containing lithium at low redshift. Consequently, data on rate coefficients for CI processes in atom - Rydberg atom collisions of Li atoms presented in Tab. 2, may be of interest for the early Universe chemistry. We therefore computed the necessary quantities.

\subsubsection{Io atmosphere}

In Brown \& Chaffee (1974) the first neutral sodium cloud near Jovian satellite Io has been detected (see also paper Brown, 1974; Fegley \& Zolotov, 2000). Since the investigation of these sodium clouds are necessary to better understand the interaction between Io's atmosphere and Jovian magnetosphere and the processes in the Jovian surroundings (Mendillo et al., 1990; Wilson et al., 2002), the data on CI processes during alkali RA collisions may be of interest. In a recent paper Schaefer \& Fegley (2005), authors estimated and expected abundance of lithium at Io.

The obtained CI rate coefficient $K^{(a b)}(n, T)$ (see Tab. 1) are calculated for a wide range of plasma parameter important for the models of Io's atmospheres (Strobel et al., 1994). CI processes may be important and could be used for accurately modelling and computer simulations. The investigation of these processes is needed for better understanding of the interaction between the Io's atmosphere and Jovian magnetosphere and of the processes in the Jovian environment. In Fig 2 the density and temperature altitude profiles for moderatedensity (on panel a) left), and high-density (on panel a) right) of Io model atmospheres (Strobel et al., 1994; Moses et al., 2002) are presented. The Fig. 2 (panels b), c), d)) shows the total rate coefficient $K_{c i}^{(a b)}(n, T)$ for CI processes in $\mathrm{Li}^{*}(n)+\mathrm{Na}, \mathrm{Li}^{*}(n)+\mathrm{Li}$ and $\mathrm{Na}^{*}(n)+\mathrm{Na}$ collisions for modeled atmospheres of Io (Strobel et al., 1994; Moses et al., 2002). For both models it can be observed the growth of coefficients at higher altitudes. Also, one can see that the rate 

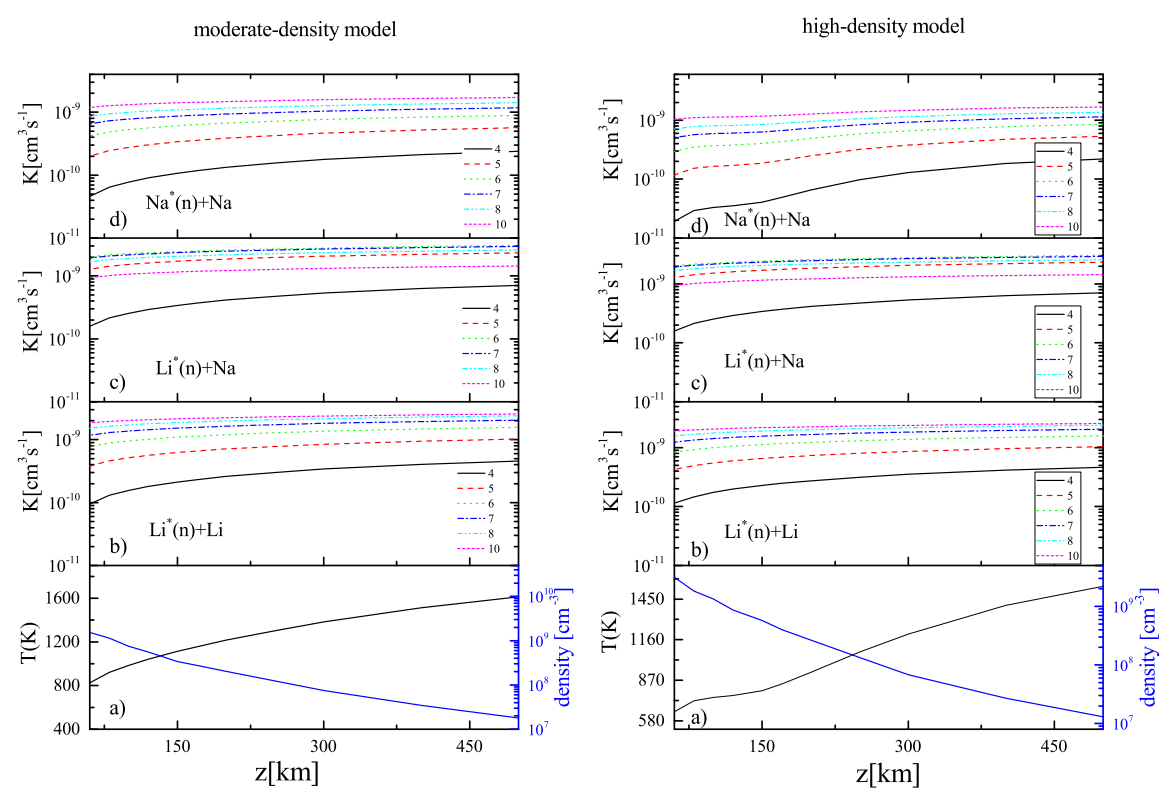

Figure 2. (left) Moderate-density model atmosphere of Io: the total rate coefficient $K_{c i}^{(a b)}(n, T)$ for CI processes in $\operatorname{Li}^{*}(n)+\mathrm{Na}, \mathrm{Li}^{*}(n)+\mathrm{Li}$ and $\mathrm{Na}^{*}(n)+\mathrm{Na}$ altitude profiles (panels b,c,d) for the corresponding model (a) (Strobel et al., 1994); (right) High-density model atmosphere of Io: the total rate coefficient $K_{c i}^{(a b)}(n, T)$ for CI processes in $\mathrm{Li}^{*}(n)+\mathrm{Na}, \mathrm{Li}^{*}(n)+\mathrm{Li}$ and $\mathrm{Na}^{*}(n)+\mathrm{Na}$ altitude profiles (panels b,c,d) for the corresponding model (a) (Strobel et al., 1994).

coefficients increase with the increase of the principal quantum number $n$ and that they are the largest for the non-symmetric collisions.

\subsubsection{Interstellar gas chemistry}

The CI processes i.e. its associative channel (1(a) and 2(a)), are responsible for the formation of molecular ions and potentially important for molecular formations in interstellar gas (Dalgarno \& Black, 1976). For knowing interstellar gas chemistry and for models of interstellar clouds the cross sections and rate coefficients for reactions (Srećković et al., 2018) are needed. The data for molecular ions $\mathrm{LiNa}^{+}, \mathrm{LiH}^{+} \mathrm{Li}_{2}^{+}$and $\mathrm{Na}_{2}^{+}$are given and analysed in this contribution. The relative contribution (probability) of the associative channels (i.e. creation of molecular ions) are presented in Fig. 3 on the example of $\mathrm{Na}^{*}(n)+\mathrm{Na}$, $\mathrm{Li}^{*}(n)+\mathrm{Na}, \mathrm{Li}^{*}(n)+\mathrm{Li}$ collisions. As expected the probability for the creation of molecular ions as CI product is noticeable and higher at lower temperatures 

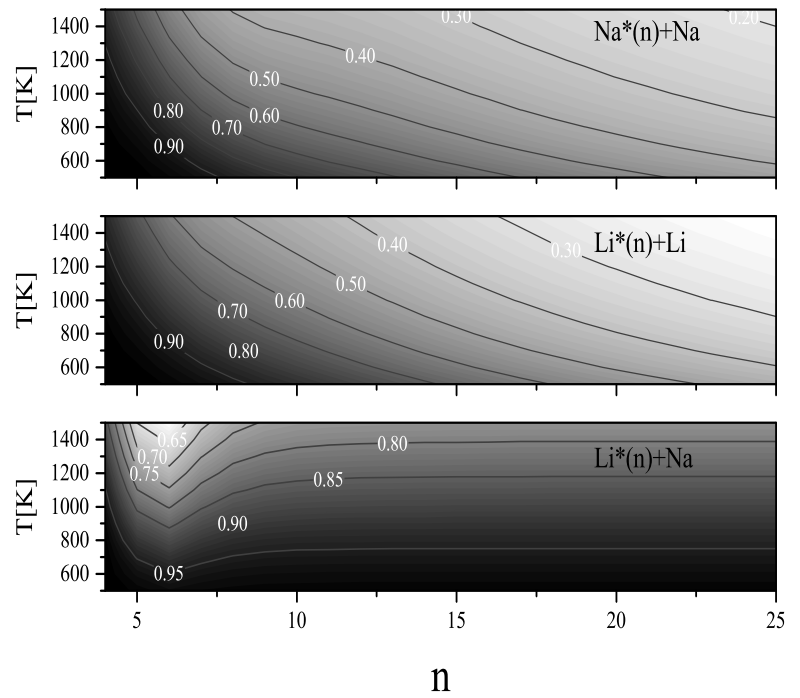

Figure 3. The surface plot of the quantity $K^{(a)}(n, T) / K^{(a b)}(n, T)$ i.e. probability of creation of molecular ions $\mathrm{A}_{2}^{+}$and $\mathrm{AB}^{+}$in $\mathrm{CI}$ collisions.

and $n$ (see Fig. 3). Unlike presented cases in $\mathrm{H}^{*}(n)+\mathrm{Li}$ collisions the associative channel is totally negligible for the analyse conditions and consequently this channel could not be the main source of the $\mathrm{LiH}^{+}$molecular ion creation.

\section{Summary}

In this paper the rate coefficients for the CI processes in $\mathrm{Li}^{*}(n)+\mathrm{Na}, \mathrm{Li}^{*}(n)+\mathrm{Li}$, $\mathrm{H}^{*}(n)+\mathrm{Li}$ and $\mathrm{Na}^{*}(n)+\mathrm{Na}$ collisions were calculated. In order to enable the better and more adequate use of data, we present the results for the rate coefficients with a simple and accurate fitting formula. The calculated data have been applied to the models of atmosphere of Io. Also, the obtained rate coefficients could be very useful for the upgrading of modelling of different weakly ionized layers in atmospheres of various stars (solar like stars, lithium stars, etc) and low temperature laboratory plasmas. Note that the presented results indicate that considered CI processes are factors which influence on the ionization degree and atom excited-state populations in weakly ionized layers of atmospheres of various stars and must have a very significant influence on the optical properties of investigated atmospheres. 
Acknowledgements. This work is made within projects of the Ministry of Education, Science and Technological Development of Serbia 176002 and III44002.

\section{References}

Beuc, R., Movre, M., \& Pichler, G., High Temperature Optical Spectra of Diatomic Molecules at Local Thermodynamic Equilibrium. 2018a, Atoms, 6, 67

Beuc, R., Peach, G., Movre, M., \& Horvatić, B., Lithium, sodium and potassium resonance lines pressure broadened by helium atoms. 2018b, Astron. Astrophys. Tran., 30, 315

Brown, R. A. \& Chaffee, Jr., F. H., High-Resolution Spectra of Sodium Emission from Io. 1974, Astrophys. J., Lett., 187, L125

Brown, R. A., Optical line emission from Io. 1974, in IAU Symposium, Vol. 65, Exploration of the Planetary System, ed. A. Woszczyk \& C. Iwaniszewska, 527-531

Chary, R., Zuckerman, B., \& Becklin, E. E., Observational constraints on the origin of metals in cool DA-type white dwarfs. 1999, in ESA Special Publication, Vol. 427, The Universe as Seen by ISO, ed. P. Cox \& M. Kessler, 289

Coppola, C. M., Lodi, L., \& Tennyson, J., Radiative cooling functions for primordial molecules. 2011, Mon. Not. R. Astron. Soc., 415, 487

Cremonese, G., Boehnhardt, H., Crovisier, J., et al., Neutral Sodium from Comet Hale-Bopp: A Third Type of Tail. 1997, Astrophys. J., Lett., 490, L199

Cremonese, G., Huebner, W. F., Rauer, H., \& Boice, D. C., Neutral sodium tails in comets. 2002, Adv. Space Res., 29, 1187

Dalgarno, A. \& Black, J. H., Molecule formation in the interstellar gas. 1976, Rep. Prog. Phys., 39, 573

Debes, J. H., Walsh, K. J., \& Stark, C., The link between planetary systems, dusty white dwarfs, and metal-polluted white dwarfs. 2012, Astrophys. J., 747, 148

Efimov, D., Bruvelis, M., Bezuglov, N., et al., Nonlinear Spectroscopy of Alkali Atoms in Cold Medium of Astrophysical Relevance. 2017, Atoms, 5, 50

Fegley, B. \& Zolotov, M. Y., Chemistry of Sodium, Potassium, and Chlorine in Volcanic Gases on Io. 2000, Icarus, 148, 193

Gnedin, Y. N., Mihajlov, A. A., Ignjatović, L. M., et al., Rydberg atoms in astrophysics. 2009, New Astron. Rev., 53, 259

Klyucharev, A. N., Bezuglov, N. N., Matveev, A. A., et al., Rate coefficients for the chemi-ionization processes in sodium- and other alkali-metal geocosmical plasmas. 2007, New Astron. Rev., 51, 547

Li, H., Aoki, W., Matsuno, T., et al., Enormous Li Enhancement Preceding Red Giant Phases in Low-mass Stars in the Milky Way Halo. 2018, Astrophys. J., Lett., 852, L31

Marinković, B. P., Jevremović, D., Srećković, V. A., et al., BEAMDB and MolDdatabases for atomic and molecular collisional and radiative processes: Belgrade nodes of VAMDC. 2017, Eur. Phys. J. D, 71, 158 
Mendillo, M., Baumgardner, J., Flynn, B., \& Hughes, W. J., The extended sodium nebula of Jupiter. 1990, Nature, 348, 312

Mihajlov, A., Ignjatović, L. M., Srećković, V., \& Djurić, Z., The influence of (n-n')mixing processes in $\mathrm{He}^{*}(\mathrm{n})+\mathrm{He}(1 \mathrm{~s} 2)$ collisions on $\mathrm{He}^{*}(\mathrm{n})$ atoms populations in weakly ionized helium plasmas. 2008, J. Quant. Spectrosc. Radiat. Transf., 109, 853

Mihajlov, A. A., Ignjatović, L. M., Srećković, V. A., \& Dimitrijević, M. S., Chemiionization in Solar Photosphere: Influence on the Hydrogen Atom Excited States Population. 2011a, Astrophys. J., Suppl., 193, 2

Mihajlov, A. A., Ignjatović, L. M., Srećković, V. A., \& Dimitrijević, M. S., The influence of chemi-ionization and recombination processes on spectral line shapes in stellar atmospheres. 2011b, Open Astron., 20, 566

Mihajlov, A., Sakan, N., Srećković, V., \& Vitel, Y., Modeling of continuous absorption of electromagnetic radiation in dense partially ionized plasmas. 2011c, J. Phys. A, 44, 095502

Mihajlov, A. A., Srećković, V. A., Ignjatović, L. M., \& Dimitrijević, M. S., AtomRydberg-atom chemi-ionization processes in solar and DB white-dwarf atmospheres in the presence of (n - n')-mixing channels. 2016, Mon. Not. R. Astron. Soc., 458, 2215

Mihajlov, A. A., Srećković, V. A., Ignjatović, L. M., \& Klyucharev, A. N., The ChemiIonization Processes in Slow Collisions of Rydberg Atoms with Ground State Atoms: Mechanism and Applications. 2012, J. Clust. Sci., 23, 47

Moses, J. I., Zolotov, M. Y., \& Fegley, B., Photochemistry of a Volcanically Driven Atmosphere on Io: Sulfur and Oxygen Species from a Pele-Type Eruption. 2002, Icarus, 156, 76

O'Keeffe, P., Bolognesi, P., Avaldi, L., et al., Experimental and theoretical study of the chemi-ionization in thermal collisions of Ne Rydberg atoms. 2012, Phys. Rev. A, 85, 052705

North, P., Polosukhina, N., Malanushenko, V., \& Hack, M., The AP star HD 83368 may be a lithium-spotted pulsator. 1998, Astron. Astrophys., 333, 644

Pichler, G., Makdisi, Y., Kokaj, J., et al., Superheating effects in line broadening of dense alkali vapors. 2017, in J. Phys. Conf. Ser., Vol. 810, Journal of Physics Conference Series, 012013

Puy, D., Dubrovich, V., Lipovka, A., Talbi, D., \& Vonlanthen, P., Molecular fluorine chemistry in the early Universe. 2007, Astron. Astrophys., 476, 685

Schaefer, L. \& Fegley, B., Alkali and halogen chemistry in volcanic gases on Io. 2005, Icarus, 173, 454

Shavrina, A. V., Polosukhina, N. S., Pavlenko, Y. V., et al., The spectrum of the roAp star HD 101065 (Przybylski's star) in the Li I $6708 \AA$ spectral region. 2003, Astron. Astrophys., 409, 707 
Shavrina, A. V., Polosukhina, N. S., Zverko, J., et al., Lithium on the surface of cool magnetic CP stars. II. Spectrum analysis of HD 83368 and HD 60435 with lithium spots. 2001, Astron. Astrophys., 372, 571

Srećković, V., Dimitrijević, M., Ignjatović, L., Bezuglov, N., \& Klyucharev, A., The Collisional Atomic Processes of Rydberg Hydrogen and Helium Atoms: Astrophysical Relevance. 2018, Galaxies, 6, 72

Srećković, V., Ignjatović, L., Jevremović, D., Vujčić, V., \& Dimitrijević, M., Radiative and Collisional Molecular Data and Virtual Laboratory Astrophysics. 2017, Atoms, $\mathbf{5}, 31$

Strobel, D. F., Zhu, X., \& Summers, M. F., On the vertical thermal structure of Io's atmosphere. 1994, Icarus, 111, 18

Vujčić, V., Jevremović, D., Mihajlov, A. A., et al., MOL-D: A Collisional Database and Web Service within the Virtual Atomic and Molecular Data Center. 2015, J. Astrophys. Astron., 36, 693

Wilson, J. K., Mendillo, M., Baumgardner, J., et al., The Dual Sources of Io's Sodium Clouds. 2002, Icarus, 157, 476

Xu, S. \& Jura, M., The Drop during Less than 300 Days of a Dusty White Dwarf's Infrared Luminosity. 2014, Astrophys. J., Lett., 792, L39 